\title{
Invasion biology and conservation biology: time to join forces to explore the links between species traits and extinction risk and invasiveness
}

\author{
Mark van Kleunen ${ }^{1 *}$ and David M. Richardson ${ }^{2}$ \\ 'Centre for Invasion Biology, School of Biological and Conservation Sciences, \\ University of KwaZulu-Natal, Private Bag X01 Scottsville, Pietermaritzburg \\ 3209, South Africa \\ ${ }^{2}$ Centre for Invasion Biology, Department of Botany and Zoology, Stellenbosch \\ University, Private Bag X1 Matieland, Stellenbosch 7602, South Africa
}

\begin{abstract}
Expansion and decline of species are natural phenomena (Levin, 2000). However, owing to the increasing influence of humans worldwide, the processes driving expansion and decline have speededupdramatically. Hence, we have the current biodiversity crisis. Humanmediated forces are making some species rarer (ie, driving them towards extinction) while at the same time making some species expand their ranges ('invasive' sensu Richardsonet al., 2000). Undisputedly, the immediate causes for extinction of native species and invasiveness of aliens are extrinsic factors, such as habitat destruction and climate change. However, the ultimate causes have to be ecological and life-history characteristics of species (Kotiaho et al., 2005). Therefore, important research directions in conservation biology and invasion biology are the analyses of species traits associated with rarity (Murray et al., 2002)
\end{abstract}

and invasiveness (Pyšek and Richardson, 2007), respectively. The first are important for prioritizing conservation efforts, and the second are important for prioritizing eradication efforts and the development of screening protocols of potential invasiveness of species considered for introduction to other regions.

Unravelling the links between species traits and extrinsic factors is highly complex, particularly because assessment of the causes of rarity and invasiveness relies mainly on retrospective analyses since large-scale controlled experiments are often not feasible (Richardson et al., 2004). However, with the increasing availability of large, widely accessible databases and analytical approaches (eg, Wilson et al., 2007), it is becoming easier to test which traits are associated with rarity and invasiveness, and under which conditions.

*Author for correspondence. Current address: Institute of Plant Sciences, University of Bern, Altenbergrain 21, CH-3013 Bern, Switzerland. Email: vkleunen@ips.unibe.ch 
To date, conservation biologists and invasion biologists have searched separately for species traits associated with rarity and invasiveness, respectively. This is surprising because the same data sets offer insights for both issues. Moreover, conservation biologists and invasion biologists encounter similar problems in their search for determinants of rarity and invasiveness, and could benefit from each other's solutions. Furthermore, one would intuitively expect rarity and invasiveness to be on opposing ends of the species trait spectrum. For example, while excessive long-distance dispersal, caused by increased fecundity in the absence of key natural enemies, is frequently a crucial factor facilitating invasion of alien species, insufficient long-distance dispersal, due to disruption of reproduction, might threaten native species (Trakhtenbrot et al., 2005). If this 'opposite-ends-of-the-spectrum' hypothesis holds for most traits, information on species attributes of rare species could be used to predict attributes of potential invaders and vice versa, when accurate data on one of these groups is lacking. Therefore, a closer collaboration between the two groups of researchers by sharing databases and methodology could benefit both research disciplines.

Reviews of studies comparing traits of rare and common plant species conclude that there is some, albeit limited, evidence for consistent predictors of plant rarity (Bevill and Louda, 1999; Murray et al., 2002). Similarly, a recent review of studies comparing invasive with either native or unsuccessful alien plant species concludes that there is some, albeit limited, evidence for consistent predictors of plant invasiveness (Pyšek and Richardson, 2007). One pattern that appears is the generally high fecundity of invasive alien species (Pyšek and Richardson, 2007) and the generally low fecundity of rare species (Murray et al., 2002). Furthermore, Pandit (2006) found that, among 1183 species in the Indian subcontinent, endangered species have fewer chromosomes than non-endangered ones, while invasive species have more chromosomes than non-invasive ones. For many other traits, however, the patterns are not clear for several reasons. Insufficient data exist for many traits, particularly those that need to be assessed experimentally (van Kleunen and Johnson, 2007). For example, only a few studies have explored how interspecific variation in phenotypic plasticity of traits is related to rarity (Murray et al., 2002) or invasiveness (Richards et al., 2006). In addition, it is difficult to compare studies directly because they apply different criteria in defining rarity and invasiveness, they often use small sample sizes, and they assess rarity or invasiveness at different spatial scales. Moreover, for studies on invasiveness, the control group differs between studies; some used native species and others used non-invasive alien species as control (Pyšek and Richardson, 2007). Although the latter approach is preferable (Hamilton et al., 2005; Muth and Pigliucci, 2006), some of these studies have not considered whether the non-invasive alien species have actually been introduced (but see van Kleunen et al., 2007).

An important distinction that most studies comparing rare and common native species do not make is the one between species that have been rare for a very long time (eg, many endemic species) and those that have become rare recently. This distinction is critical because species of the first group might have (evolved) traits that allow them to persist in small populations and prevent them from going extinct (Rabinowitz, 1981; Gaston, 1994; Gaston and Kunin, 1997). Species of the other group may have become rare recently owing to human-mediated habitat changes to some extent because they lack such traits. Interestingly, this could explain why some species that are rare in their native range (and have been so prior to human-mediated disturbance) can spread rapidly from small founder populations when introduced to new areas. Clearly, the evolutionary history of some species has equipped them for conquests as aliens. For example, many pine 
species that spread rapidly from small refugia after historical periods of glaciation are now invasive in other parts of the world (Richardson and Rejmánek, 2004). When one does not distinguish between species that have been rare for a long time and the ones that have become rare recently, it might be impossible to find attributes associated with species rarity.

Although apparent discrepancies among studies may be artifacts of different definitions of invasiveness and rarity and the lack of control for the role of extrinsic factors, it may also indicate that predictors of rarity and invasiveness are highly context-specific (Alpert et al., 2000). Therefore, when framed in an objective, biogeographical context, one might expect life-history traits to be more strongly associated with invasiveness (but see Hamilton et al., 2005) and rarity. Moreover, when considered in the same biogeographical context, one might find endangered native and invasive alien plants to be at opposite ends of the species traits spectrum.

Insufficient information is available to enable us to conclude that rarity and invasiveness of plants can be predicted from species attributes and that they involve the same set of traits. If the latter is true, both conservation biologists and invasion biologists would profit because data on traits of threatened rare species could be used to predict the fate of species introduced to new areas and vice versa. A better understanding of the correlates of humaninduced rarity in native species might also benefit control programmes against invasive alien species whose aim is usually to make the species rare or, ideally, (locally) extinct (Panetta, 2007). Therefore, we suggest that conservation and invasion biologists should combine forces to improve our understanding of the links between species attributes and rarity and invasiveness. Collaboration by sharing databases and methodology will benefit both research fields.

\section{Acknowledgements}

We thank the DST-NRFCentre of Excellence for Invasion Biology for financial support.

\section{References}

Alpert, P., Bone, E. and Holzapfel, C. 2000 Invasiveness, invasibility and the role of environmental stress in the spread of non-native plants. Perspectives in Plant Ecology, Evolution and Systematics 3, 52-66.

Bevill, R.L. and Louda, S.M. 1999: Comparisons of related rare and common species in the study of plant rarity. Conservation Biology 13, 493-98.

Gaston, K.J. 1994: Rarity. London: Chapman and Hall.

Gaston, K.J. and Kunin, W.E. 1997: Rare-common differences: an overview. In Kuning, W.E. and Gaston, K.J., editors, The biology of rarity: causes and consequences of rare-common differences, London: Chapman and Hall, 12-29.

Hamilton, M.A., Murray, B.R., Cadotte, M.W., Hose, G.C., Baker, A.C., Harris, C.J. and Licari, D. 2005: Life-history correlates of plant invasiveness at regional and continental scales. Ecology Letters 8, 1066-74.

Kotiaho, J.S., Kaitala, V., Komonen, A. and Päivinen, J. 2005: Predicting the risk of extinction from shared ecological characteristics. Proceedings of National Academy of Sciences 102, 1963-67.

Levin, D.A. 2000: The origin, expansion, and demise of plant species. New York: Oxford University Press.

Murray, B.R., Thrall, P.H, Gill, A.M. and Nicotra, A.B. 2002: How plant life-history and ecological traits relate to species rarity and commonness at varying spatial scales. Austral Ecology 27, 291-310.

Muth, N.Z. and Pigliucci, M. 2006: Traits of invasives reconsidered: phenotypic comparisons of introduced invasive and introduced non-invasive plant species within two closely related clades. American Journal of Botany 93, 188-96.

Pandit, M.K. 2006: Continuing the search for pattern among rare plants: are diploid species more likely to be rare? Evolutionary Ecology Research 8, 543-52.

Panetta, F.D. 2007: Evaluation of weed eradication programs: containment and extirpation. Diversity and Distributions 13, 33-41.

Pyšek, P. and Richardson, D.M. 2007: Traits associated with invasiveness in alien plants: where do we stand? In Nentwig, W., editor, Biological invasions, ecological studies 193, Berlin: Springer-Verlag, 97-126.

Rabinowitz, D. 1981: Seven forms of rarity. In Synge, H., editor, The biological aspects of rare plant conservation, Chichester: Wiley, 205-17.

Richards, C.L., Bossdorf, O., Muth, N.Z., Gurevitch, J. and Pigliucci, M. 2006: Jack of all trades, master of some? On the role of phenotypic plasticity in plant invasions. Ecology Letters 9, 981-93.

Richardson, D.M. and Rejmánek, M. 2004: Conifers as invasive aliens: a global survey and predictive framework. Diversity and Distributions 10, 321-31. 
Richardson, D.M., Pyšek, P., Rejmánek, M. Barbour, M.G., Panetta, F.D. and West, C.J. 2000: Naturalization and invasion of alien plants: concepts and definitions. Diversity and Distributions 6, 93-107.

Richardson, D.M., Rouget, M. and Rejmánek, M. 2004: Using natural experiments in the study of alien tree invasions: opportunities and limitations. In Gordon, M.S. and Bartol, S.M., editors, Experimental approaches to conservation biology, Berkeley, CA: University of California Press, 180-201.

Trakhtenbrot, A., Nathan, R., Perry, G. and Richardson, D.M. 2005: The importance of longdistance dispersal in biodiversity conservation. Diversity and Distributions 11, 173-81. van Kleunen, M. and Johnson, S.D. 2007: South African Iridaceae with rapid and profuse seedling emergence are more likely to become naturalized in other regions. Journal of Ecology 674-81.

van Kleunen, M., Johnson, S.D. and Fischer, M. 2007: Predicting naturalization of southern African Iridaceae in other regions. Journal of Applied Ecology 594-603.

Wilson, J.R.U., Richardson, D.M., Rouget, M., Procheş, Ş. Amis, M.A., Henderson, L. and Thuiller, W. 2007: Residence time and potential range: crucial considerations in modelling plant invasions. Diversity and Distributions 13, 11-22. 